\title{
A dynamic model and performance analysis of a stepped rotary flow control valve
}

\author{
Karem Abuowda ${ }^{1}$, Siamak Noroozi ${ }^{1}$, Mihai Dupac ${ }^{1}$, and Phil \\ Godfry $^{2}$
}

\begin{abstract}
The hydraulic independent metering (IM) is an advanced actuator driving technique that allows the implementation of advanced control algorithms or methods. The main concept of IM is to control hydraulic actuators ports, which are the meterin and meter-out, separately. In this paper, a novel stepped rotary type valve has been developed for embedding in hydraulic independent metering systems, instead of conventional types such as poppet and spool. The insertion leads to developing different and novel control techniques, which require a software in loop and hardware in loop simulation of the proposed system. The paper explores the dynamic representation of this valve and defines its own performance limitations. This includes the development of a linear model comprising its two main sub-parts which are the stepper motor and the rotary orifice. Consequently, the linear timeinvariant methods are used to explore the performance of the valve by considering the effect of different parameters namely the pressure drop, friction coefficient, damping coefficient and bristle coefficient.
\end{abstract}

\section{Keywords}

Linear dynamic modeling, Stepped rotary valve, Root locus analysis, Hydraulic system control, Independent Metering, Software in loop simulation

\section{Nomenclature}

$\Delta \theta \quad$ The change of the angle

degree

$\lambda \quad$ The interval between the stepper motor stator poles winding

$m$

${ }^{1}$ Bournemouth University, UK

${ }^{2}$ Hydreco Hydraulic Ltd, UK

Prepared using sagej.cls [Version: 2015/06/09 v1.01] 
$\theta \quad$ Flow jet angle

degree

$\theta_{i} \quad$ The initial stepper motor rotor position

degree

$B \quad$ Stepper motor viscous friction constant

Nms/rad

D Stepper motor viscous damping coefficient

$i_{a} \quad$ Stepper motor coil $A$ current

$i_{b} \quad$ Stepper motor coil $B$ current

$J \quad$ Stepper motor inertia

$K g \cdot m^{2}$

$K_{m} \quad$ Stepper motor detent torque constant

$L \quad$ The length of the spool located inside the sleeve

$n \quad$ The number of the balancing grooves

$N_{r} \quad$ Stepper motor number of teeth

$R \quad$ Stepper motor coil resistance

$T_{L} \quad$ Stepper motor Total load torque

$N m$

$V_{a} \quad$ Stepper motor coil $A$ supplied voltage $\quad V$

$V_{b} \quad$ Stepper motor coil $B$ supplied voltage $\quad V$

$w \quad$ The width of the balancing grooves

$Z \quad$ The deflection average of the asperities on two contacting surfaces

$\Delta p \quad$ Pressure difference $\quad P a$

$\omega \quad$ Angular velocity $\mathrm{rad} / \mathrm{sec}$

$\omega_{s} \quad$ Stribick characteristics velocity $\mathrm{m} / \mathrm{s}$

$\begin{array}{lll}\sigma_{0} & \text { Stiffness coefficient } \quad N / m\end{array}$

$\sigma_{1} \quad$ Damping coefficient $\quad \mathrm{Ns} / \mathrm{m}$

$\begin{array}{lll}\sigma_{2} & \text { Friction coefficient } \quad \mathrm{Ns} / \mathrm{m}\end{array}$

$A_{o} \quad$ Opening area $m^{2}$

$A_{\text {sp.op }}$ Spool opening area $\quad m^{2}$

$C_{c} \quad$ Contraction coefficient

$C_{v} \quad$ Velocity Coefficient

$R_{e . s p} \quad$ External spool radius $m$ 
$R_{e . s p} \quad$ Internal spool radius $\quad m$

$T_{c} \quad$ Columb friction $N$

$\begin{array}{lll}T_{s} & \text { Static friction } & N\end{array}$

$T_{\text {st.fl }}$ Steady state flow torque $\mathrm{Nm}$

$T_{t r . f l}$ Transient flow torque $\quad \mathrm{Nm}$

\section{Introduction}

Hydraulic drives are heavily used for a variety of applications ranging from industrial, military, aerospace, and earthmoving machines due to their unique and valuable characteristics. They have high load capabilities and high power to weight ratio ${ }^{1}$. However, these drives still suffer from some deficiencies which are energy losses and poor controllability. Many systems developed to overcome these shortcomings ${ }^{2}$ can be separated into load-sensing, digital hydraulics, hybrid systems and independent metering ${ }^{3}$.

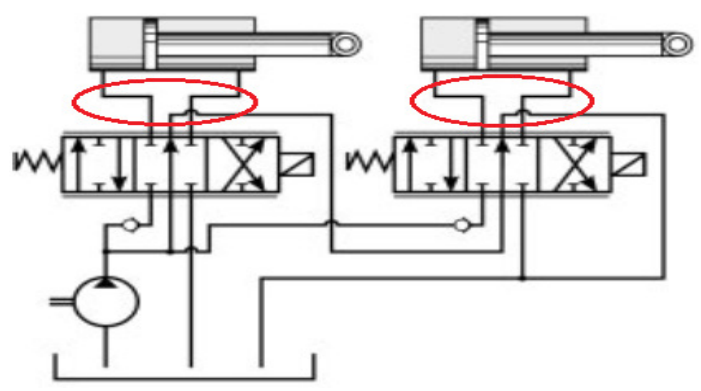

Figure 1. Traditional Open center system ${ }^{4}$

Regarding independent metering, this technique is based on individual control of hydraulic actuators ports. In conventional drive control, the spool valve mechanically connects the meter-in and the meter-out as shown in the red circles in Figure 1. Traditional valves cause the controller to be blind about one of the cylinder chamber pressure which reduces the controllability ${ }^{4}$. Moreover, it increases energy losses by preventing energy regeneration. Using individual metering allows the controller to independently control the two chamber pressures as illustrated in Figure 2, and this allows energy regeneration between the actutor chambers and the fluid lines. Energy regeneration modes were studied and analyzed by Shenouda ${ }^{5}$. Different types of control valves were used to implement IM. The poppet or programmable valve is such an important type ${ }^{6 ; 7}$. This type of valves uses a solenoid as an actuator, and suffers from some controllability drawbacks as discussed by ${ }^{8 ; 9}$. On the other hand, a rotary valve has been developed to be implemented in high flow rate applications. This valve is proposed to be used for IM system ${ }^{10}$, Figure 3 . The dynamic performance analysis is required in order to design an advanced control system.

Prepared using sagej.cls 


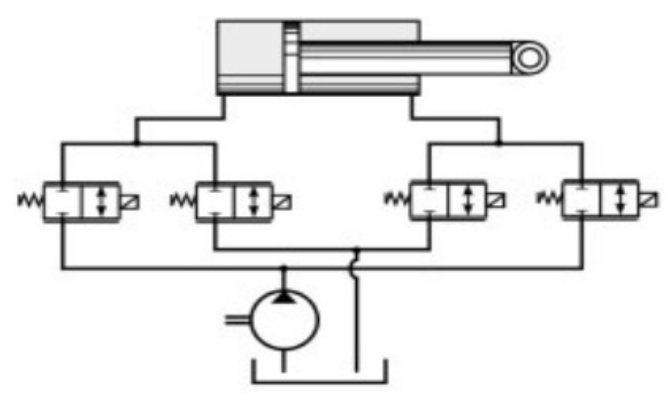

Figure 2. Individual metering control system ${ }^{4}$

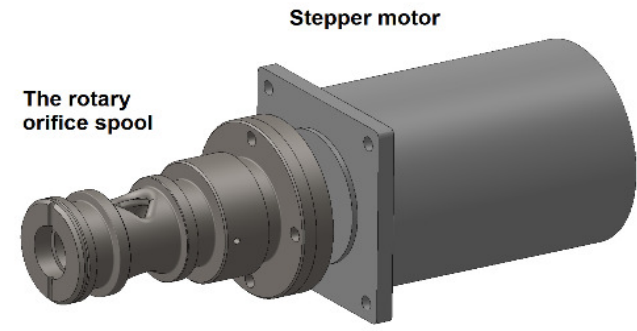

Figure 3. Stepped rotary flow control valve

In the literature review, there is a surprising number of research about modeling and analysis of flow control valves such as traditional spool valve and poppet valve. As this valve will be used instead of the poppet one, this review will target the methods which were used with a poppet valve. Also, it will consider the subparts of the new valve, which are the rotary orifice and the stepper motor. Generally, few examples of research included electrical and mechanical parts of the system, and this will be explored during this research. Firstly the poppet valve, it was developed by HUSCO international to implement independent metering control method form ${ }^{11}$. The poppet valve is known by difficulties in modeling because of its nonlinear characteristics ${ }^{12}$. An Easy5 model of the cartridge valve was developed to be used in system simulation ${ }^{9}$. A linear, nonlinear model and model validation for a two-stage electrohydraulic valve, Valvistor, were investigated ${ }^{8}$. A performance analysis of two-stage poppet valve was performed using root locus analysis for a linear model. The investigations were performed by applying different operation conditions similar to what conducted in ${ }^{13}$ and $^{14}$. Regarding rotary parts, a vane actuator linear and nonlinear models were developed. These models involved introducing a Coulomb friction due to seals, and the linear model was used for initial parameters estimations ${ }^{15}$ and ${ }^{16}$. A three-way electrohydraulic rotary valve driven by a DC motor was designed and mathematically modeled $^{17}$. The research included a mathematical modeling of the main parameters acting during operations. These parameters are viscous friction torque, seal friction 
torque, steady-state and transient flow torque. The steady flow torque was deeply analyzed for servo-operated rotary directional valve ${ }^{18}$. The stepper motor has been widely used for different applications due to its valuable characteristics. The main types with their constructions of the stepper motor were discussed by ${ }^{19}$. A dynamical simulation of the stepper motor ${ }^{20}$. A position control of PM stepper motor using exact linearization technique was evaluated ${ }^{21}$. On the other hand, the coupling between the mechanical, electrical and fluid parts of the valve was evaluated by some researchers. For example, the solenoid current, fluid flow, and the mechanical parts coupling were explained in ${ }^{22}$. The effect of friction on stepper motor in robotics applications was modeled and evaluated ${ }^{23}$. In this paper, a linear model for a new stepped rotary valve was developed.The model combines the two parts of the valve which are the stepper motor and the rotary orifice. Using the model, a root locus method was adopted to determine its performance limitations to be used during simulation and future developments of the system. Further, an initial step response analysis is performed.

\section{Mathematical modeling of the valve}

This stepped rotary flow control valve consists of two main parts which are the stepper motor and the mechanical rotary orifice illustrated in Figure 4. The coupling between these two subsystems requires the stepper motor to overcome the torques generated from the mechanical part. The steady state flow torque in equation (6), the transient flow torque in equation (12) and the friction torque in equation (14) have been developed and evaluated using ${ }^{10},{ }^{24},{ }^{25}$, and ${ }^{26}$. Likewise, the equations representing the stepper motor are included in the state space representation in equation (18). The steady state flow torque acting on the rotary spool from the both orifices (Figure $4 \mathrm{~b}$ ) was investigated in $^{10}$

$$
T_{s t . f l}=C_{v} Q_{t} \sqrt{2 \Delta p \rho} R_{e . s p} \sin \theta
$$

where $T_{s t . f l}$ is the steady state flow, $C_{v}$ is the velocity coefficient, $Q_{t}$ is the flow rate, $\Delta p$ is the pressure difference, $\theta$ is the flow jet angle, $\rho$ is the oil density and $R_{e . s p}$ is the external spool radius, The magnitude of the velocity coefficient depends on the contraction coefficient of the sharp-edged orifice $C_{c}$ according to a following relation

$$
C_{v}=1 / \sqrt{1-C_{c}^{2}}
$$

The contraction coefficient in its turn specifies a ratio of a cross sectional area of a compressed flow in a vena contracta $A_{v c}$ over the orifice area $A_{o}$. The coefficient is a function of the geometry of the outlet ${ }^{27}$

$$
C_{c}=\frac{A_{v c}}{A_{o}}
$$

The discharge coefficient is a function of the contraction coefficient as well as the velocity coefficient:

$$
C_{d}=C_{v} C_{c}=\frac{C_{c}}{\sqrt{1-C_{c}^{2}}}
$$

Prepared using sagej.cls 
Thus, the Bernoulli equation expressed through the contraction coefficient takes form

$$
Q_{t}=\frac{C_{c} A_{o}}{\sqrt{1-C_{c}^{2}}} \sqrt{\frac{2 \Delta p}{\rho}}
$$

Replacing equations (2), (3), (4), and (5) into equation ( 1 ), one can obtain

$$
T_{s t . f l}=\frac{2 C_{c} \Delta p A_{o} R_{e . s p} \sin \theta}{1-C_{c}{ }^{2}}
$$

where $T_{s t . f l}$ is the steady state flow, $C_{c}$ is the contraction coefficient, $\Delta p$ is the pressure difference, $A_{o}$ is the opening area, and $R_{e . s p}$ is the external spool radius,

In the sliding spool valves, the transient flow force is proportional to a spool velocity and a rate of pressures changes acting on a small fluid element inside the control volume. It's applicable for rotary valves as well with substitution of linear motion to angular one ${ }^{24}$. However, in the hollow rotary spool, the pressure changes in down and up stream channels don't cause a pressure difference on the edges of the rotary orifice. This doesn't lead the formation of neither a tangential force nor torque on the spool. Hence, the only contributor to the transient flow torque is the fluid inertial component, which can be determined through a moment of inertia $I_{f l}$ of a fluid volume and a spool angular acceleration $\gamma$

$$
T_{t r . f l}=I_{f l} \gamma=I_{f l} \frac{d \omega}{d t}
$$

The moment of inertia of the fluid body (hollow cylinder or tube) along its longitudinal axis corresponding to the valve main axis is equal to ${ }^{28}$

$$
I_{f l}=m_{f l} \frac{\left(R_{e . s p}^{2}+R_{i . s p}^{2}\right)}{2}
$$

where $R_{e . s p}$ is the external radii of the spool, $R_{i . s p}$ is the internal radii of the spool, total fluid mass $m_{f l}$ in both orifices under acceleration. This mass $m_{f l}$ is found through the total volume of tubular control elements $V_{f l}$ in both orifices. Each of control volumes represents a portion of a hollow cylinder, which is located within an angle $\alpha$, covered by the spool window.

$$
m_{f l}=V_{f l} \rho=\frac{2 \Pi \alpha\left(R_{e . s p}^{2}-R_{i . s p}^{2}\right) h \rho}{360^{\circ}}
$$

The height of the fluid cylinder $h$ is variable along a central arc in symmetry plane of the opening. Effectively the fluid cylinder height is equal to the width of the window. Thus, it can be expressed through an equivalent area $A_{s p . e q}$, which equates to the spool single window opening area $A_{\text {sp.op }}$. Hence, referring to Figure 5, the sought height $h$ is a ratio of the spool opening $A_{s p . o p}$ to the central axis arc length $l$ :

$$
\begin{aligned}
& A_{\text {sp.op }}=A_{\text {sp.eq }}=h l, \\
& h=A_{\text {sp.eq }} / l=\frac{360^{\circ} A_{s p . o p}}{2 \Pi \alpha R_{e . s p}}
\end{aligned}
$$

Prepared using sagej.cls 
Replacing equations (8 - 11) into equation (7), one can obtain

$$
T_{t r . f l}=\frac{\left(R_{e . s p}^{4}-R_{i . s p}^{4}\right) A_{s p . o p} \rho}{R_{e . s p}} \frac{d \omega}{d t}
$$

where $T_{t r . f l}$ is the transient flow torque, $R_{e . s p}$ is the external spool radius, $R_{i . s p}$ is the internal spool radius, $\omega$ is the angular velocity, $A_{s p . o p}$ is the spool opening area.

The friction torque is represented by equations (13) and $(14)^{26}$,

$$
T_{f} r=\sigma_{0}+\sigma_{1} \frac{d Z}{d t}+\sigma_{2} \omega
$$

where $\sigma_{0}$ is the stiffness coefficient, $\sigma_{1}$ is the damping coefficient, $\sigma_{2}$ is the viscous friction coefficient, and $\omega$ is the angular velocity.

$$
\frac{d Z}{d t}=\omega-\frac{\sigma_{0}|\omega|}{T_{c}+\left(T_{s}-T_{c}\right) \exp -\left(\frac{\omega}{\omega_{s}}\right)^{2}} Z
$$

where $T_{c}$ is the columb friction, $T_{s}$ is the static friction, $\omega_{s}$ is the stribick characterstics velocity, and $Z$ is the deflection average of the asperities on two contacting surfaces. Assuming the spool and the sleeve are concentric parts with a small radial clearance between them; the viscous frictional torque acting on the spool from the annular liquid volume at motion in the clearance can be considered as laminar Couette flow and calculated according to the Newtons law of viscosity:

$$
\sigma_{2} \omega=\tau A_{s p} R_{e . s p}=\frac{\mu R_{e . s p}^{2} A_{s p} \omega}{\delta}
$$

where $\tau$ is shear stress, $\mu$ the dynamic viscosity coefficient of the fluid, $A_{s p}$ the total area of the spool external cylinder subjected to the shear stress in the annual gap with a clearance

$$
\delta=R_{i . s l}-R_{e . s p}
$$

where $R_{i . s l}$ and $R_{e . s p}$ are the inner sleeve and the external spool radii respectively. Viscous shear stress $\tau$ acts on the spool cylindrical surfaces in the annular gap with the clearance $\delta$. The spool external cylinder houses two sets of balancing grooves, which prevent formation of a hydraulic lock and centre the spool concentrically inside the sleeve ${ }^{24}$, as well as two throttling orifices. These regions do not contribute to the viscous friction torque on the spool since the radial distance from the spool to the sleeve there is not equal to the clearance $\delta$. Thus, the total area of interest is

$$
A_{s p}=2 \Pi R_{e . s p}(L-n w)-2 A_{s p, o p}
$$

where $L$ is the length of the spool located inside the sleeve, $\mathrm{n}$ is the number of the balancing grooves, and $w$ is the width of the balancing grooves. The solid-to-solid sliding Coulomb friction in the case of the considered design takes place between the spool and elastomer O-ring seals and back-up rings. Sealing between moving mechanical parts is ensured by squeezed elastomer and plastic back-up rings during

Prepared using sagej.cls 
assembly. This squeeze produces the drag friction torque from an elastomer ring on the sealant part $^{25}$.

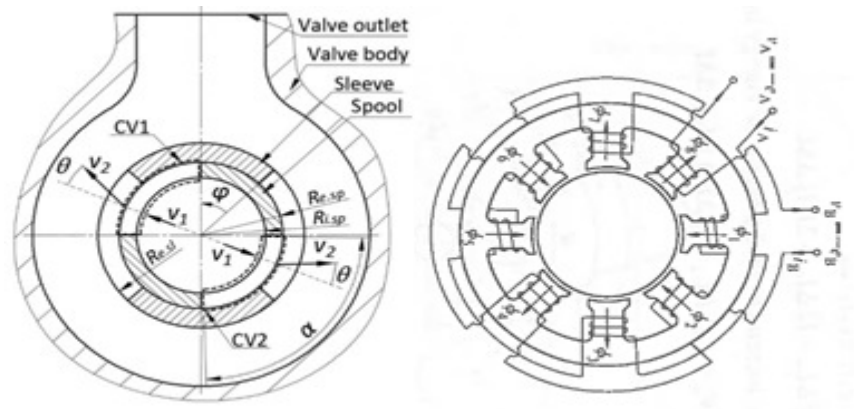

Figure 4. Schematic diagram of the vale two main parts which are the mechanical orifice and the stepper motor respectively

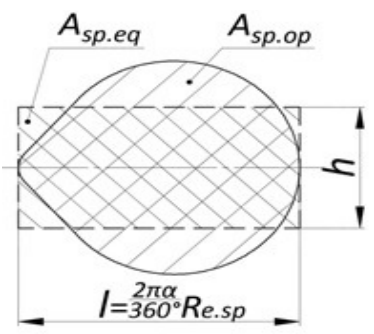

Figure 5. The mean width of the window

The stepper motor model is given by ${ }^{20 ; 29}$

$$
\left[\begin{array}{c}
\frac{d \theta}{d t} \\
\frac{d \omega}{d t} \\
\frac{d i_{a}}{d t} \\
\frac{d i_{b}}{d t}
\end{array}\right]=\left[\begin{array}{c}
\omega \\
\frac{1}{J}\left[-K_{m} i_{a} \sin \left(N_{r} \theta\right)+K_{m} i_{b} \cos \left(N_{r} \theta\right)-B \omega-T_{L}\right] \\
\frac{1}{L}\left[V_{a}-R i_{a}+K_{m} \sin \left(N_{r} \theta\right)\right] \\
\frac{1}{L}\left[V_{b}-R i_{b}-K_{m} \cos \left(N_{r} \theta\right)\right]
\end{array}\right]
$$

where $\omega$ is the angular velocity, $J$ is the inertia, $K_{m}$ is the detent torque constant, $N_{r}$ is the number of teeth, $R$ is the resistance, $T_{L}$ is the total load torque, $B$ is the viscous friction constant, $i_{a}$ is the coil $A$ current, $i_{b}$ is the coil $B$ current, $V_{a}$ is the coil $A$ supplied voltage, and $V_{b}$ is the coil $B$ supplied voltage.

Prepared using sagej.cls 


\section{Torque testing and analysis}

The friction model performance (Figure 6) based on equations (6), (12), (13), and (14) has the highest value that affects the coupling between the valve components. The torque was validated using the test rig shown in Figure 7 and 8. The test rig schematic diagram is shown in Figure 9. The testing procedure starts by fixing the pressure drop around the valve using the pump controlled speed and the pressure relief valve which is used to define the inlet pressure of the valve. Then, the valve opening is adjusted to measure the produced flow, while the torque transducer is used to measure the total applied torque on the stepper motor. The applied torque includes the steady state flow torque, the transient flow torque, and the friction torque. The independent metering technique relies on pressure compensater to reduce the complexity of the control system ${ }^{30}$, so the test was performed with pressure differences $0.25 \mathrm{MPa}, 0.75$ $\mathrm{MPa}$ and $1 \mathrm{MPa}$.

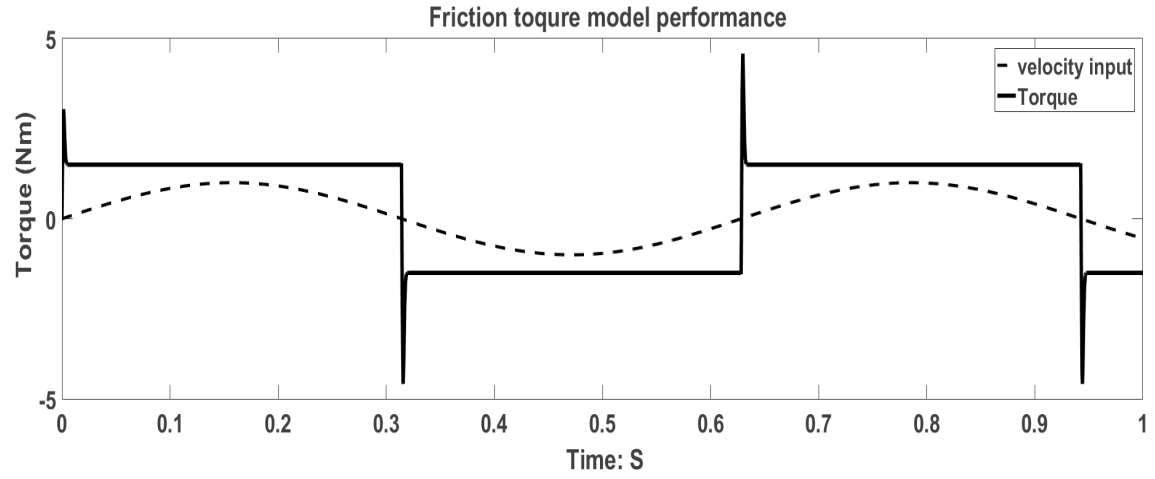

Figure 6. The simulated friction of the orifice part

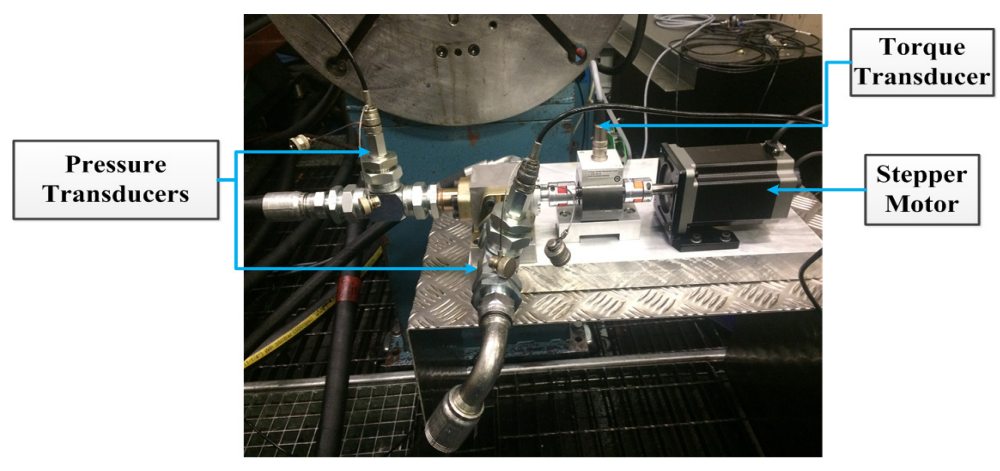

Figure 7. The test rig of the valve which contains the transducers and the stepper motor 


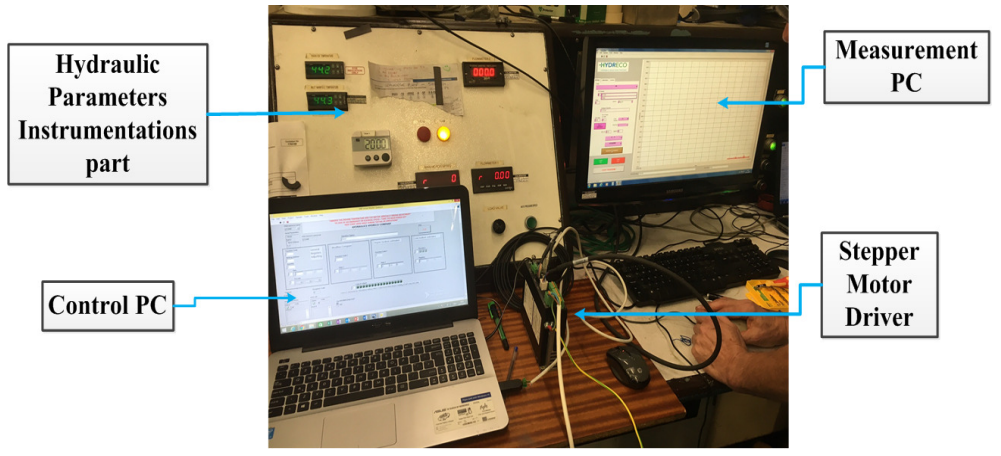

Figure 8. The main control panel which contains a driving system and measurement system

The main tools used in the test are:

- Pressure transducers - this is a Gems 3100B0040G01B000RS pressure transducer (40 bar, and 4 to $20 m A$ output).

- Valve Main Flowmeter - this is a 3100 gear motor calibrated against a VC10 Kracht flow meter.

- Leakage flow meter - this is Kracht VC1 calibrated against the VC10 meter.

- Supply pump - this is a hydraulic gear pump $160 \mathrm{cc} / \mathrm{rev}$ pump (part number 058304) in the R6 Q series from HYDRECO.

- Oil Temperature sensor - this is a $\mathrm{K}$ type thermocouple.

- Latptop - this is an Acer machine used to activate the stepper motor driver by sending commands through Modbus cable using LabView Code.

- Workstation - this is a computer connected with the measurement components and with the analogue control panel.

- Stepper motor - this is an Orientalmotor RKII series.

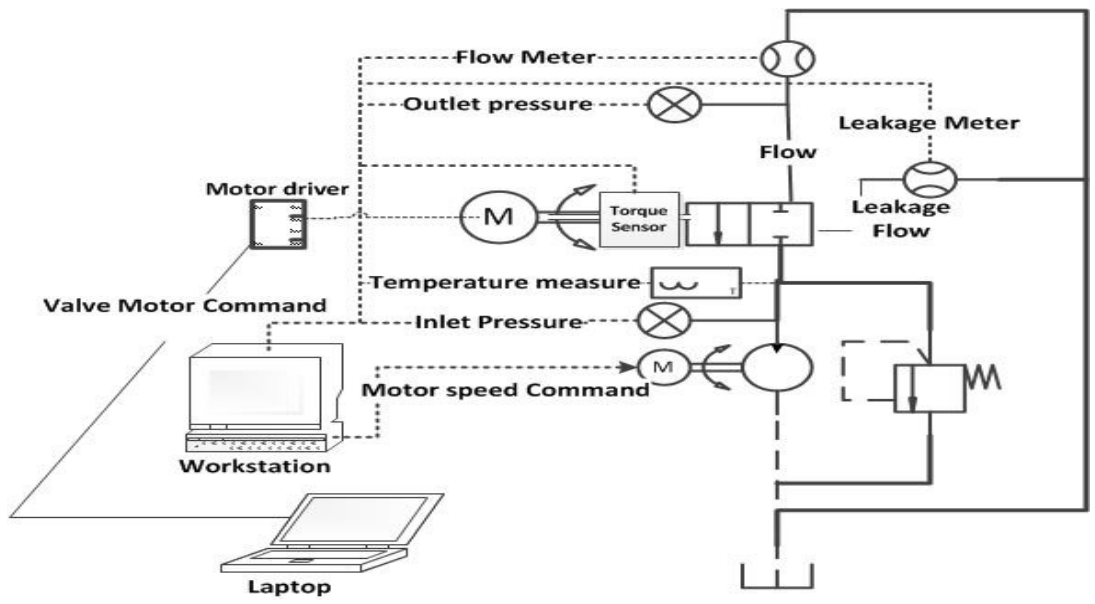

Figure 9. The schematic diagram of the test rig 
Figure 10 shows the produced torque at an opening range between $20^{\circ}$ to $90^{\circ}$ with the produced flow detected using the flow meter. One of the instant dynamic response of produced torque was measured for an opening valve of $45^{\circ}$ and $200 \mathrm{kHz}$ stepper motor switching frequency (Figure 11). These results are similar to the friction model (Figure 6) which represents the highest value of toque that affect the stepper motor performance.

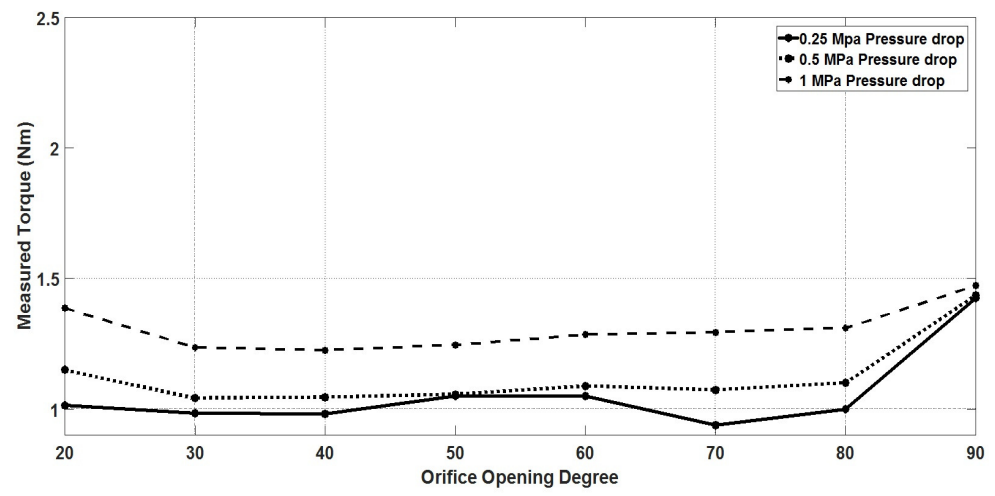

Figure 10. The produced torque from the valve with different pressure drops

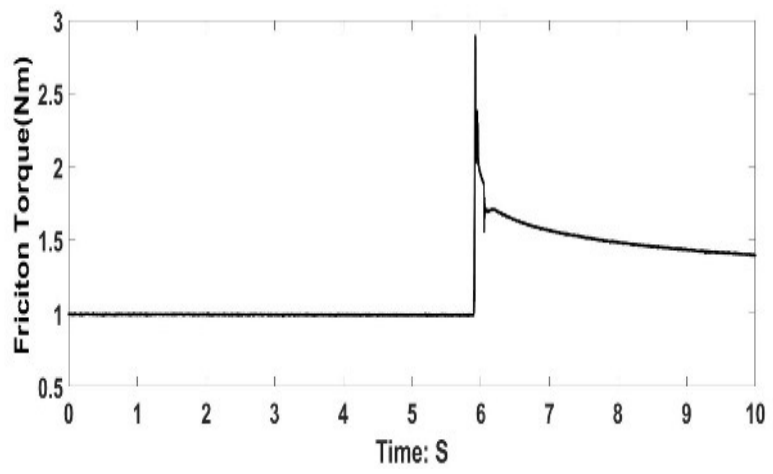

Figure 11. The instant response of the spool friction

\section{State space model linearization}

The state space representation of the valve is used to describe the system dynamics. The main equations of this representation are,

$$
\begin{aligned}
& \dot{X}(t)=A(t) X(t)+B(t) U(t) \\
& Y(t)=C(t) X(t)+D(t) U(t)
\end{aligned}
$$

The proposed stepped valve flow curve is shown in Figure (12). A polynomial equation to represent the curve using in has been obtained using curve regression, that 
is

$$
0.0173 x^{2}-0.4883 x-3.5
$$

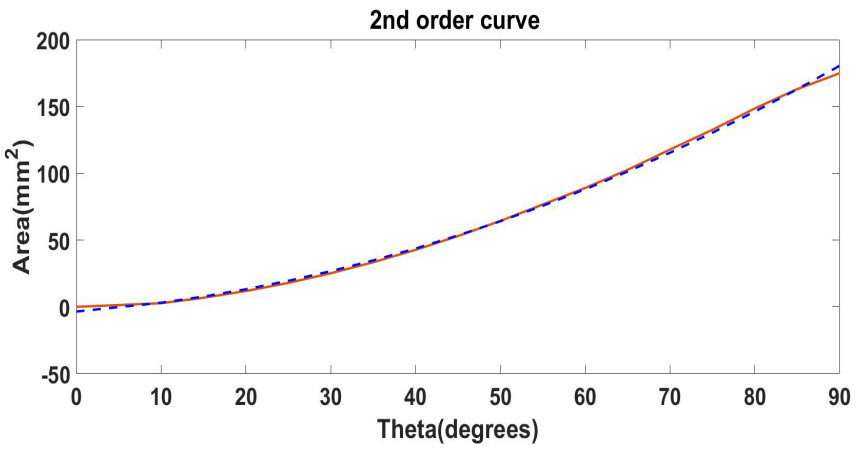

Figure 12. The curve of area v.s. rotated angle of the proposed stepped rotary valve and a second order polynomial fitting.

As the discharge coefficient is an important factor in Bernoulli equation representing the performance of the valve, a linearization point was selected based on the discharge coefficient graph of the valve shown in Figure 13. The coefficient effect starts from $10^{\circ}$ opening which is the selected point of linearization, since by selecting a $0^{\circ}$ detection may eliminate important parameters during analysis.

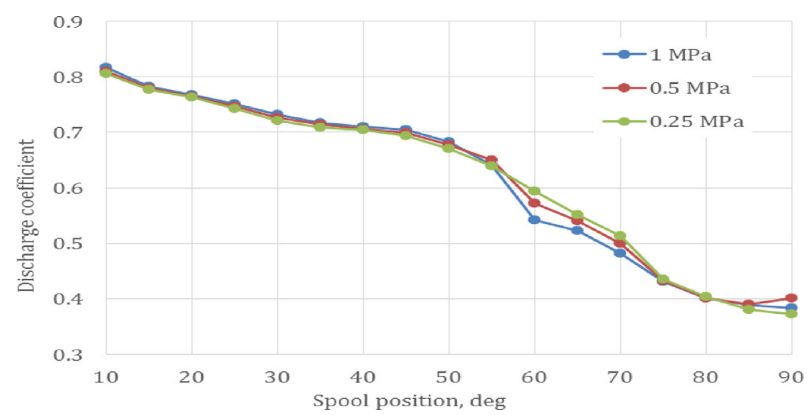

Figure 13. The discharge coefficient performance of the valve ${ }^{10}$

As previously mentioned, the transient flow torque in equation 12 is very small compared to the rest of the parameters, and this can be eliminated, resulting a model representation based only on equations (6),(13) and (18). Rearranging the equations one can obtain

Prepared using sagej.cls 


$$
\left[\begin{array}{c}
\frac{d \theta}{d t} \\
\frac{d \omega}{d t} \\
\frac{d i_{a}}{d t} \\
\frac{d i_{b}}{d t} \\
\frac{d Z}{d t} \\
\frac{d T_{c}}{d t}
\end{array}\right]=\left[\begin{array}{c}
\omega \\
\frac{1}{J}\left[-K_{m} i_{a} \sin \left(N_{r} \theta\right)+K_{m} i_{b} \cos \left(N_{r} \theta\right)-B \omega-T_{L}\right] \\
\frac{1}{L}\left[v_{a}-R i_{a}+K_{m} \sin N_{r} \theta\right] \\
\frac{1}{L}\left[v_{b}-R i_{b}-K_{m} \cos N_{r} \theta\right] \\
\omega-\frac{\sigma_{0}|\omega|}{T_{c}+\left(T_{s}-T_{c}\right) \exp -\left(\frac{\omega}{\omega_{s}}\right)^{2}} Z \\
0
\end{array}\right]\left[\begin{array}{c}
\theta \\
\omega \\
i_{a} \\
i_{b} \\
Z \\
T_{c}
\end{array}\right]
$$

By adding equations (6) and (13), one can represent the total torque in equation (22) by

$$
T_{L}=T_{f r}+T_{s t . f l}=\sigma_{0} z+\sigma_{1} \dot{z}+\sigma_{2} \omega+\frac{2 C_{c} \Delta p A_{o} R_{e . s p} \sin \theta}{1-C_{c}{ }^{2}}
$$

In a matrix form the equation (22), reads $\dot{X}=A(t) X(t)$, where the state vector is the right hand column $\mathrm{X}(\mathrm{t})$ and state matrix is the middle side $\mathrm{A}(\mathrm{t})$. The nonlinear parts of the state matrix are $K_{m} i_{a} \sin \left(N_{r} \theta\right), K_{m} i_{b} \cos \left(N_{r} \theta\right), K_{m} \sin \left(N_{r} \theta\right), K_{m} \cos \left(N_{r} \theta\right)$, $\omega-\frac{\sigma_{0}|\omega|}{T_{c}+\left(T_{s}-T_{c}\right) \exp -\left(\frac{\omega}{\omega_{s}}\right)^{2}} z$, and $A_{o}$

To linearize these parts, a Taylor series is used ${ }^{31}$, that is

$$
F(X, U)=A_{o}+A_{1} \delta X+B_{1} \delta U+\epsilon(X, U)
$$

where the linearization of the flow curve in equation (21) is given by

$$
3 \theta-27
$$

Since the operating point is around $10^{\circ}$ from equation (23) and (25) one can obtain the flow torque as

$$
T_{L}=T_{f r}+T_{s t . f l}=\sigma_{0} z+\sigma_{1} \dot{z}+\sigma_{2} \omega+\frac{2 C_{c} \Delta p(3 \theta-27) R_{e . s p} 0.2}{1-0.8^{2}}
$$

The linearization of equation $\omega-\frac{\sigma_{0}|\omega|}{T_{c}+\left(T_{s}-T_{c}\right) \exp -\left(\frac{\omega}{\omega_{s}}\right)^{2}} z$ represented by equation (27) can be performed in two forms when the valve is accelerating or settles at any point. The first form with the valve stopped at the opening is $\theta=10^{\circ}$, $T_{c}=2, T_{s}=0, \omega=0$ and $\omega_{s}=0.001$. The linearization is represented by equation (28). 


$$
\begin{aligned}
& F\left(\omega, T_{c}, T_{s}, z, \omega_{s}\right)=F\left(\omega_{0}, T_{c 0}, T_{s 0}, \omega_{s 0}, z_{0}\right)+F_{\omega}\left(\omega_{0}, T_{c 0}, T_{s 0}, \omega_{s 0}, z_{0}\right)\left(\omega-\omega_{0}\right) \\
& +F_{T c}\left(\omega_{0}, T_{c 0}, T_{s 0}, s 0, z_{0}\right)\left(T_{c}-T_{c 0}\right)+F_{T s}\left(\omega_{0}, T_{c 0}, T_{s 0}, s 0, z_{0}\right)\left(T_{s}-T_{s 0}\right) \\
& +F_{\omega_{s}}\left(\omega_{0}, T_{c 0}, T_{s 0}, \omega_{s 0}, z_{0}\right)\left(\omega_{s}-\omega_{s 0}\right)+F_{z}\left(\omega_{0}, T_{c 0}, T_{s 0}, \omega_{s 0}, z_{0}\right)\left(z-z_{0}\right)
\end{aligned}
$$

$$
F\left(\omega, T_{c}, T_{s}, z, \omega_{s}\right)=\omega
$$

The stiction friction based on Coulomb friction model was analyzed in ${ }^{25}$ by considering a linear velocity. Converting the linear velocity into angular velocity using $\omega=v / r$ where $\mathrm{r}$ is the external radius of the spool, leads to the initial values of the term $\omega_{0}=31.25, T_{c 0}=2, T_{s 0}=0, z=0$, and $\omega_{s}=0.001$,

$$
F\left(\omega, T_{c}, T_{s}, Z, \omega_{s}\right)=26.5-21.716 \sigma_{0}+\omega\left(\frac{\sigma_{0}-2}{2}\right)+\frac{125 \sigma_{0} T_{c}}{16}-\frac{125 \sigma_{0} z}{8}
$$

The linearization of the term $K_{m} i_{a} \sin \left(N_{r} \theta\right)$ can be written as

$$
\begin{aligned}
& F\left(i_{a}, \theta\right)=F\left(i_{a 0}, \theta_{0}\right)+F_{i a}\left(i_{a 0}, \theta_{0}\right)\left(i_{a}-i_{a 0}\right)+F_{\theta}\left(i_{a 0}, \theta_{0}\right)\left(\theta-\theta_{0}\right) \\
& =112.5 K_{m} \theta-1125 K_{m}
\end{aligned}
$$

The linearization of the term $K_{m} i_{b} \cos \left(N_{r} \theta\right)$ is

$$
\begin{aligned}
& F\left(i_{b}, \theta\right)=F\left(i_{b 0}, \theta_{0}\right)+F_{i b}\left(i_{b 0}, \theta_{0}\right)\left(i_{b}-i_{b 0}\right)+F_{\theta}\left(i_{b 0}, \theta_{0}\right)\left(\theta-\theta_{0}\right) \\
& =0.5624 K_{m} i_{b}
\end{aligned}
$$

The linearization of the term $K_{m} \sin \left(N_{r} \theta\right)$ is represented by

$$
561 K_{m}+56.24 K_{m} \theta
$$

The linearization of $K_{m} \cos \left(N_{r} \theta\right)$ is given by

$$
821 K_{m}-82.69 K_{m} \theta
$$

Then the linearized dynamic system is as follow

Prepared using sagej.cls 


$$
\left[\begin{array}{r}
\frac{d \theta}{d t} \\
\frac{d \omega}{d t} \\
\frac{d i_{a}}{d t} \\
\frac{d i_{b}}{d t} \\
\frac{d Z}{d t} \\
\frac{d T_{c}}{d t}
\end{array}\right]=\left[\begin{array}{c}
\omega \\
\frac{1}{J}\left[-0.829 K_{m} i_{a}+0.5624 K_{m} i_{b}-112.5 K_{m} \theta+1126.6 K_{m}\right. \\
-B \omega-\frac{\omega \sigma_{0} \sigma_{1}}{2} \omega \sigma_{0}-\sigma_{2} \omega- \\
\left.\frac{125 \sigma_{0} \sigma_{1} T_{c}}{16}+\frac{125 \sigma_{0} \sigma_{1} z}{8}-0.025 \Delta p \theta+0.225 \Delta p\right] \\
\frac{1}{L}\left[V_{a}-R i_{a}+561 K_{m}+56.24 K_{m} \theta\right] \\
\frac{1}{L}\left[V_{b}-R i_{b}-821 K_{m}-82.369 K_{m} \theta\right] \\
62.5-21.716 \sigma_{0}+\omega \frac{\sigma_{0}-2}{2}+\frac{125 \sigma_{0} T_{c}}{16}-\frac{125 \sigma_{0} Z}{8} \\
0
\end{array}\right]\left[\begin{array}{c}
\theta \\
\omega \\
i_{a} \\
i_{b} \\
Z \\
\\
T_{c}
\end{array}\right]
$$

The main matrices A, B, and C used in this study are presented in in the Appendeix.

\section{Performance analysis}

A Matlab code was developed to find the system poles distribution represented by eigenvalues of the matrix $A+B U$. Using a loop inside the code, parameter value variations shows the change of the poles distribution. The limit of pressure difference that can be applied to this valve is computed by changing the value of the dp in $\mathrm{U}$ the vector which represents the input of the state space representation. Increasing the pressure difference up to $10 \mathrm{MPa}$, as shown in Figure 14, the pole distribution shows two straight lines parallel to the $X$-axis meaning that the system is very stable during the increase in pressure, and the system damping is fixed. The poles around the origin are still to the left of the $Y$-axis meaning the valve keeps its own stability. Increasing the pressure difference, up to $20 \mathrm{MPa}$, forces the poles poles to move toward the $Y$ axis while the system keeps stability and damping as shown in Figure 15. When the pressure difference is located to point between $35 \mathrm{MPa}$ to $37 \mathrm{MPa}$ the valve start losing stability, and is hardly controllable as shown in Figure 16.

Prepared using sagej.cls 


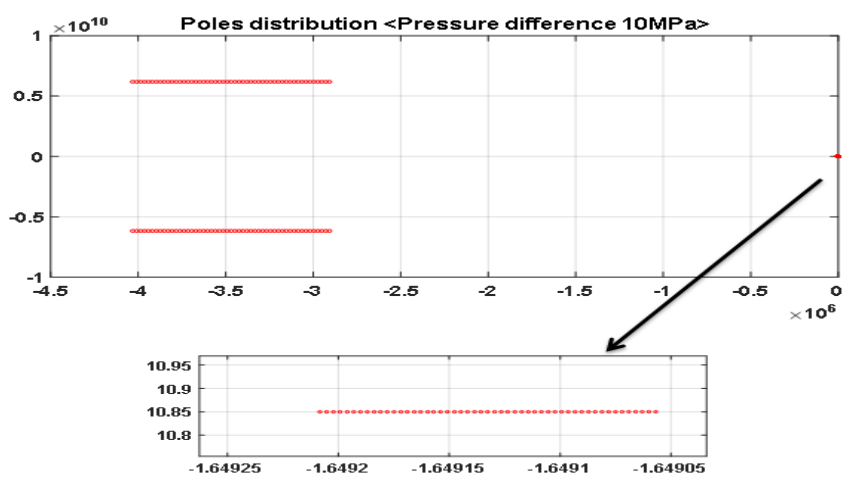

Figure 14. The poles distribution when applying pressure difference upto $10 \mathrm{MPa}$

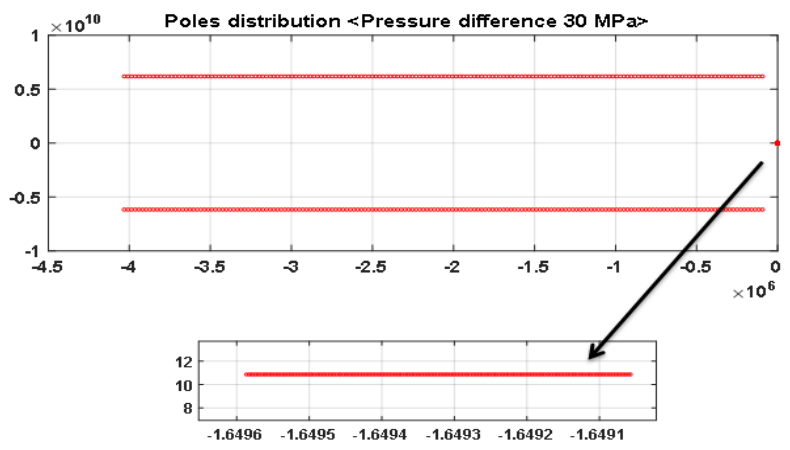

Figure 15. The poles distribution when applying pressure difference upto $20 \mathrm{MPa}$

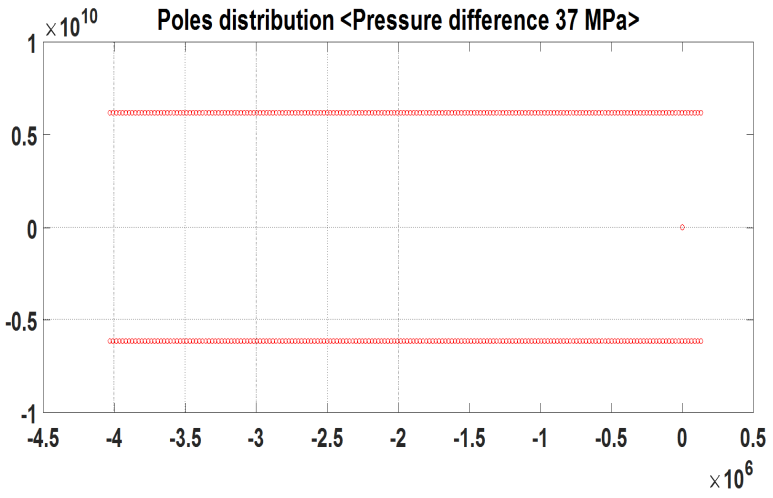

Figure 16. The poles distribution when applying pressure difference upto $37 \mathrm{MPa}$ 
The second critical parameter during the design process, especially related to the seal material is the stiffness coefficient. It has been noticed that an increase in stiffness is improving the valve stability. Initially, the stiffness coefficient was selected to be $1 e 5$ $\mathrm{N} / \mathrm{m}$ as shown in Figure 17. By increasing the coefficient upto $1 e 7 \mathrm{~N} / \mathrm{m}$ the poles start to leave the area around the origin, which is critical, and move back to left side to be distributed along the $X$-axis, Figure 18. As shown in Figure 19, it can be seen that the system has the best stability when the stiffness value increased to $1 e 8 \mathrm{~N} / \mathrm{m}$.

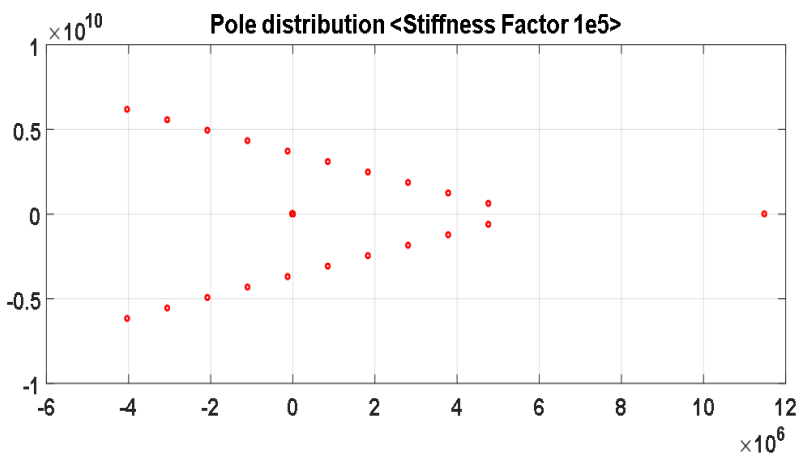

Figure 17. The poles distribution by changing the stiffness coefficient to $1 e 5$

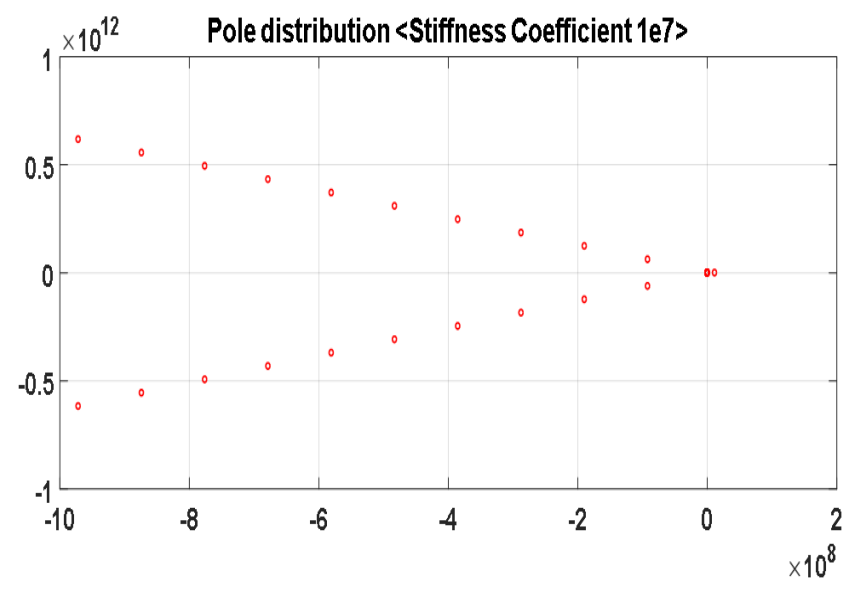

Figure 18. The poles distribution by changing the stiffness coefficient to $1 e 7$ 


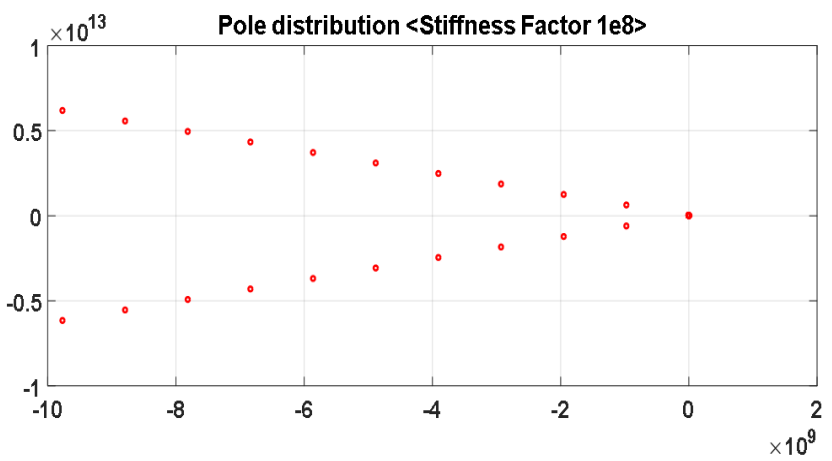

Figure 19. The poles distribution by changing the stiffness coefficient to $1 e 8$

The model damping coefficient effect is illustrated for the poles moving away from the $X$-axis toward the right side as shown in Figure 20. The increase in the friction coefficient is shown in Figure 21. As indicated in the figure, there is a great similarity in performance between the $d p$ and the friction coefficient. The correlation between these two factors agrees with the results obtained during testing, Figure 10.

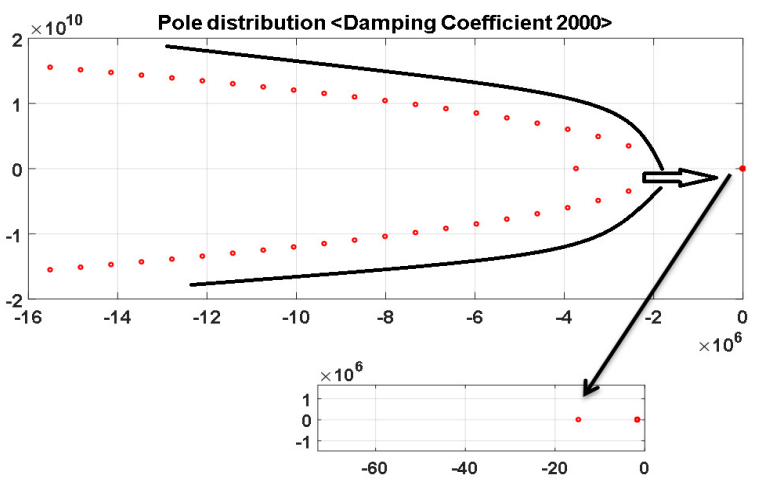

Figure 20. The pole distribution due to damping coefficient change upto 2000 


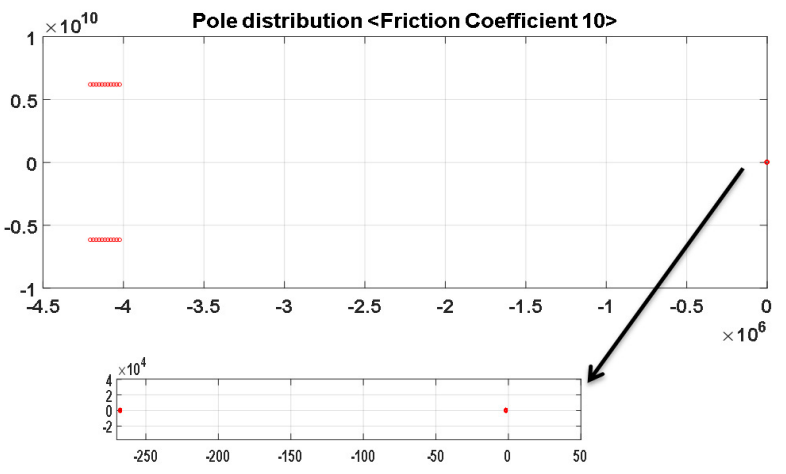

Figure 21. The pole distribution due to friction coefficient change upto 10

Since the stepper motor of the valve that is directly coupled to the orifice spool (Figure 3), the dynamic performance of the spool can be represented by the dynamic performance of the stepper motor rotor. The behaviour of a range of stepper motors have been analyzed and tested in many studies ${ }^{19 ; 32}$ while neglecting the external loads.For this study, the load torque represents an important parameter a especially since the friction is a factor of angular speed. Therefore, a similar procedure as in ${ }^{19 ; 32}$ is performed while considering the average value of the torque load. The transfer function obtained for the valve is represented in equations (35)-(37).

$$
\begin{gathered}
G(s)=\frac{\Delta \theta}{\theta_{i}}=\frac{s^{2}+\left(\frac{R}{L}+\frac{(D+T)}{J}\right) s+\left(\frac{R(D+T)}{L J}+K_{p} \omega_{n p}^{2}\right)}{s^{3}+\left(\frac{R}{L}+\frac{(D+T)}{J}\right) s^{2}+\left(\frac{R(D+T)}{L J}+\left(K_{p}+1\right)\right) s+\left(\frac{R}{L}\right) \omega_{n p}^{2}} \\
K_{P}=\frac{K_{m} \sin ^{2}\left(\frac{N_{r} \lambda}{2}\right)}{N_{r} L I_{o} \cos \left(\frac{N_{r} \lambda}{2}\right)} \\
\omega_{n p}^{2}=\frac{2 N_{r} L I_{o} \cos \left(\frac{N_{r} \lambda}{2}\right)}{J}
\end{gathered}
$$

where $\Delta \theta$ is the angle variation, $\theta_{i}$ is the initial stepper motor rotor position, $D$ is the viscous damping coefficient, $\lambda$ is the interval between the stator poles winding. As shown in Figure 22, the response rise time is $0.698 \mathrm{~s}$, the overshoot is $42 \%$, the settling time is $6.59 \mathrm{~s}$, and the settling value is 0.77 .

Prepared using sagej.cls 


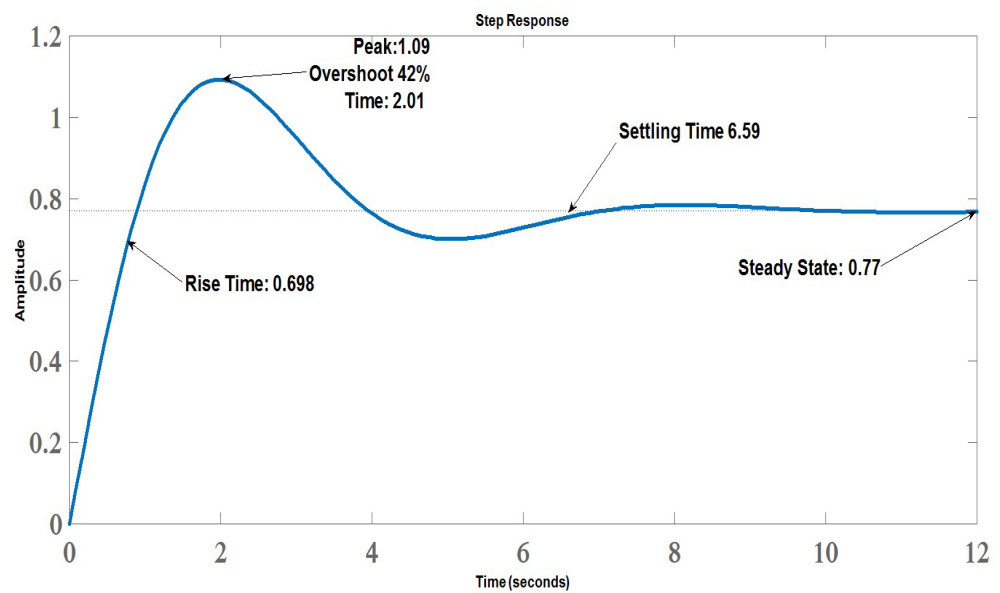

Figure 22. The step response of the system

\section{Conclusion}

In this paper, the dynamic model of a novel stepped rotary control valve has been developed. Root locus analysis was used to determine this design stability and the performance limitations due to its parameter variations. In the analysis, different coefficients namely the pressure difference, friction coefficient, damping coefficient, and bristle coefficient have been considered for deign and control process. The effect of these parameter variations illustrates that the applied pressure difference could be up to $35 \mathrm{MPa}$ while the valve keeps stability. On the other hand, increasing the bristle coefficient improve the valve performance. The other parameter which is the damping coefficient should be highly increased to stop the poles move toward the right side of the origin and this reduces the system stability. Friction coefficient follows the same performance of the pressure difference which indicate the expected relationship between the pressure difference effect and the friction coefficient during design. The single step response analysis shows an overshoot up to $42 \%$ and a slow rise and settling time.

\section{References}

1. Parr A. Hydraulics and pneumatics: a technician's and engineer's guide. Elsevier, 2011.

2. Mattila J, Koivumäki J, Caldwell DG et al. A survey on control of hydraulic robotic manipulators with projection to future trends. IEEE/ASME Transactions on Mechatronics 2017; 22(2): 669-680.

3. Murrenhoff H, Sgro S and Vukovic M. An overview of energy saving architectures for mobile applications. In Proceedings of the 9th International Fluid Power Conference, Aachen, Germany. pp. 24-26.

4. Eriksson B and Palmberg JO. Individual metering fluid power systems: challenges and opportunities. Proceedings of the institution of mechanical engineers, part I: journal of systems and control engineering 2011; 225(2): 196-211.

Prepared using sagej.cls 
5. Shenouda A. Quasi-static hydraulic control systems and energy savings potential using independent metering four-valve assembly configuration. $\mathrm{PhD}$ Thesis, Georgia Institute of Technology, 2006.

6. Opdenbosch P, Sadegh N, Book W et al. Auto-calibration based control for independent metering of hydraulic actuators. In Robotics and Automation (ICRA), 2011 IEEE International Conference on. IEEE, pp. 153-158.

7. Eriksson B. Mobile fluid power systems design: with a focus on energy efficiency. $\mathrm{PhD}$ Thesis, Linköping University Electronic Press, 2010.

8. Zhang R, Alleyne AG and Prasetiawan EA. Performance limitations of a class of two-stage electro-hydraulic flow valves. International Journal of Fluid Power 2002; 3(1): 47-53.

9. Liu S, Krutz G and Yao B. Easy5 model of two position solenoid operated cartridge valve. In ASME 2002 International Mechanical Engineering Congress and Exposition. American Society of Mechanical Engineers, pp. 63-67.

10. Okhotnikov I, Noroozi S, Sewell P et al. Evaluation of steady flow torques and pressure losses in a rotary flow control valve by means of computational fluid dynamics. International Journal of Heat and Fluid Flow 2017; 64: 89-102.

11. Tabor KA. Velocity based method of controlling an electrohydraulic proportional control valve, 2004. US Patent 6,775,974.

12. Opdenbosch P, Sadegh N, Book W et al. Modelling an electro-hydraulic poppet valve. International Journal of Fluid Power 2009; 10(1): 7-15.

13. Muller MT and Fales RC. Design and analysis of a two-stage poppet valve for flow control. International Journal of Fluid Power 2008; 9(1): 17-26.

14. Fales R. Stability and performance analysis of a metering poppet valve. International Journal of Fluid Power 2006; 7(2): 11-17.

15. Yao J, Jiao Z, Ma D et al. High-accuracy tracking control of hydraulic rotary actuators with modeling uncertainties. IEEE/ASME Transactions on Mechatronics 2014; 19(2): 633-641.

16. La Hera PM, Mettin U, Westerberg $S$ et al. Modeling and control of hydraulic rotary actuators used in forestry cranes. In Robotics and Automation, 2009. ICRA'09. IEEE International Conference on. IEEE, pp. 1315-1320.

17. Yang Y, Guglielmino E, Dai JS et al. Modeling of a novel 3-way rotary type electrohydraulic valve. In Information and Automation (ICIA), 2010 IEEE International Conference on. IEEE, pp. 1463-1468.

18. Wang H, Gong G, Zhou H et al. Steady flow torques in a servo motor operated rotary directional control valve. Energy Conversion and Management 2016; 112: 1-10.

19. Kenjo T and Sugawara A. Stepping motors and their microprocessor controls. Clarendon Press Oxford, 1994.

20. Kepiński R, Awrejcewicz J and Lewandowski D. Dynamical simulation of a nonlinear stepper motor system. International Journal of Dynamics and Control 2015; 3(1): 31-35.

21. Nollet F, Floquet $\mathrm{T}$ and Perruquetti W. Observer-based second order sliding mode control laws for stepper motors. Control engineering practice 2008; 16(4): 429-443.

22. Meng F, Zhang H, Cao D et al. System modeling, coupling analysis, and experimental validation of a proportional pressure valve with pulsewidth modulation control. IEEE/ASME Transactions on Mechatronics 2016; 21(3): 1742-1753.

23. Konowrocki R, Szolc T, Pochanke A et al. An influence of the stepping motor control and friction models on precise positioning of the complex mechanical system. Mechanical Systems and Signal Processing 2016; 70: 397-413.

Prepared using sagej.cls 
24. Jelali M and Kroll A. Hydraulic servo-systems: modelling, identification and control. Springer Science \& Business Media, 2012.

25. Bisztray-Balku S. Tribology of hydraulic seals for alternating motion. Periodica Polytechnica Engineering Mechanical Engineering 1995; 39(3-4): 225.

26. Pennestrì E, Rossi V, Salvini $\mathrm{P}$ et al. Review and comparison of dry friction force models. Nonlinear dynamics 2016; 83(4): 1785-1801.

27. Gerhart PM, Gerhart AL and Hochstein JI. Munson, Young and Okiishi's Fundamentals of Fluid Mechanics, Binder Ready Version. John Wiley \& Sons, 2016.

28. Budynas RG, Nisbett JK et al. Shigley's mechanical engineering design, volume 8. McGraw-Hill New York, 2008.

29. Bendjedia M, Ait-Amirat Y, Walther B et al. Sensorless control of hybrid stepper motor. In Power Electronics and Applications, 2007 European Conference on. IEEE, pp. 1-10.

30. Sitte A and Weber J. Structural design of independent metering control systems. In 13th Scandinavian International Conference on Fluid Power; June 3-5; 2013; Linköping; Sweden. 092, Linköping University Electronic Press, pp. 261-270.

31. Oaks OJ and Cook G. Piecewise linear control of nonlinear systems. IEEE Transactions on Industrial Electronics and Control Instrumentation 1976; 23(1): 56-63.

32. Ellis P. Analysis and control of the permanent-magnet stepper motor. Radio and Electronic Engineer 1971; 41(7): 302-308.

\section{Appendix}

The matrices of the state space main equations $A, U$ and $B$ are:

$$
\begin{aligned}
& A=\left[\begin{array}{cccccc}
0 & 1 & 0 & 0 & 0 & 0 \\
\frac{-11.25 K_{m}-0.025 \Delta p}{J} & \frac{-B-\frac{\sigma_{0} \sigma_{1}}{2}+\sigma_{1}-\sigma_{2}}{J} & \frac{-0.829 K-m}{J} & \frac{0.5624 K_{m}}{J} & \frac{125 \sigma_{0} \sigma_{1}}{8} & \frac{-125 \sigma_{0} \sigma_{1}}{16} \\
\frac{56.24 K_{m}}{L} & 0 & \frac{-R}{L} & 0 & 0 & 0 \\
\frac{98 K_{m}}{L} & 0 & \frac{-R}{L} & 0 & 0 & 0 \\
0 & \frac{\sigma_{0}-2}{2} & 0 & 0 & \frac{-125 \sigma_{0}}{8} & \frac{+125 \sigma_{0}}{16} \\
0 & 0 & 0 & 0 & 0 & 0
\end{array}\right]
\end{aligned}
$$

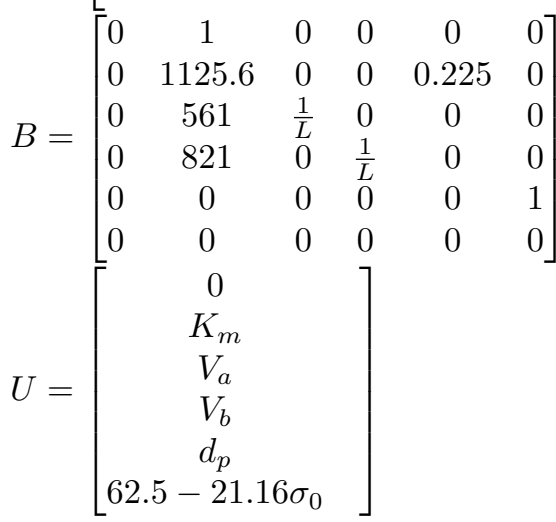

Prepared using sagej.cls 